\title{
EMULATION OF POD CURVES FROM SYNTHETIC DATA OF PHASED ARRAY ULTRASOUND TESTING
}

\author{
P. Hammersberg ${ }^{1}$, G. Persson ${ }^{1}$, and H. Wirdelius ${ }^{1}$ \\ ${ }^{1}$ Department of Materials and Manufacturing Technology \\ Chalmers University of Technology, 41296 Gothenburg, Sweden
}

\begin{abstract}
Reliability of non-destructive techniques (NDT) is traditionally quantified by experimental determined probability of detection (POD). Such multi-parameter variability studies require a significant amount of experiments for each application verified, making them extremely costly. Reducing the need of experimental data by simulations requires both a verified simulation model and a procedure for effective computer experiments. Here synthetic POD curves for ultrasonic phased array testing have been generated using simSUNDT for signal amplitude simulations and a procedure to emulate response variability by predictive (meta) modeling and Monte Carlo simulations.
\end{abstract}

Keywords: Ultrasonic, Phased Array, Synthetic-POD, Computer Experiments, Meta-Modeling

PACS: $81.70 . \mathrm{Cv}, 43.35 . \mathrm{Zc}$

\section{INTRODUCTION}

There are three basic perspectives on the risk for catastrophic failure of a system: likelihood of failure, process capability $\left(\mathrm{C}_{\mathrm{pk}}\right)$ and probability of detection (POD). The former can be interpret as - if there is a flaw, how dangerous is it relative size and location. Process capability, on the other hand, refers to how often such dangerous flaw occurs. The POD captures the chances of finding a dangerous flaw before catastrophic failure. These three concepts relate directly to the risk priority number (RPN) from the commonly used failure-mode-effect-analysis (FMEA) [1] where it is used to prioritize risks and direct interdisciplinary development resources to what is most important. RPN is the product of a three classifications: severity times occurrence times detectability. One of the great benefits with FMEA is that it challenges the three engineering communities of product development, manufacturing and quality surveillance to express system risks with commonly understood system parameters, that otherwise easily tend to be fragmented. The driving force behind the present work is to explore the problem to express NDT capabilities in the interdisciplinary context by exploring the opportunities and pitfalls with synthetically produced POD.

The evaluation of the performance of a certain NDT procedure, containing the object to be tested, equipment and operating instructions, normally requires an extensive number of measurements on samples with different flaw sizes, before the probability to detect a certain flaw size (a) can be estimated. Since a NDT system contain many sources of variation the same flaw may once be detected and once not, depending of the variation 
of all parameters affecting the procedure. The total variation seen is the sum of variation from many sources, whereas the contribution from variations of the defect should be large compared to the rest for well performing systems. The contribution from equipment and procedure is generally referred to as system repeatability and the contribution from operators as measurement system reproducibility. However, since the number of parameters influencing grow very quickly and in many cases are difficult to keep under control and to estimate, POD results tend to be valid only for very specific inspection procedure. All identifiable parameters involved are subdivided into influential and essential as according to [2]:

- Influential parameters can potentially influence the outcome of an inspection

- Essential parameters are those influential parameters that essentially alters the outcome of the inspection

POD bring a lot of valuable information of a NDT systems limitation, but is on the other hand very expensive to generate experimentally and therefore severely limits the number of influential factors to include in the studies. This also limits the possibilities to easily relate NDT performance to other overlaying system engineering, manufacturing and quality parameters.

\section{$\underline{\text { Aim and Problem }}$}

The aim here is to develop a methodology to emulate synthetic POD for parameter studies. The context is a novel testing procedure for phased array ultrasonic inspection. And the method is predictive modeling (meta-modeling) of a verified simulation environment with Monte Carlo simulated signal amplitude distributions as base for POD generation. Even if it is possible to simulate the phased array output signal with high accuracy, it will not be practical to generate data for wider parameter studies of POD, since the simulation run time, with this specific software, is relatively long, 5-10 min per data point. The purpose is to show that it is possible to emulate relevant synthetic POD, with calculation times only a fraction of a second per data point. It also significantly improves the possibilities for parameter studies and system understanding build-up without a detailed mathematical modeling of all variation sources in the NDT system. The overall aim is to reduce cost of parameter studies in order to facilitate NDE and NDT applicability.

\section{Procedure of Synthetic POD in Short}

The procedure to generate synthetic POD is based on a second order predictive model of the transfer function of the system that relates input parameter settings to the output response of the system. The predictive model, or meta-model, is in this case emulated with designed computer experiments with a verified simulation model of the phased array ultrasonic probe. The simulation model calculates the signal amplitude from side-drilled holes of varying diameter and depth, at different settings of sample and equipment parameters. The output distribution of the signal response, that forms the base for the POD value, is then generated with Monte Carlo simulations of the input parameter variations through the predictive model. 


\section{NDT Capability by POD}

Experimentally determined POD is a well-known procedure described, for example, in the MIL-HDBK-1823A [3], ENIQ report No 41 [4] or "NDE Reliability Data Analysis" by A.P. Berens in Metals Handbook, Vol. 17 [5]. Figure 1 illustrates the principle even though this figure shows synthetic POD. For each defect size and depth, in this case, a number of experimental runs capture the output signal variation from a set of essential input parameters. The number of runs grows very quickly with the number of essential parameters to investigate, making experimentally captured POD tedious and expensive. Practically this means that some assumptions need to done. To keep the amount of practical experimentation down the recommended procedure in (MIL-HDBK-1823A 2009 ) is to use at least 40 samples with varying defect size (a) in the interesting range. And with at least one measure (â) of every defect size and the assumption that scatter of â is the same in the full range of the defect size (a), the POD(a) can be estimated. In Fig. 2, Monte Carlo simulated distributions of the output signal amplitude from the simulations of the phased array system in Fig. 5 indicate that the assumption of constant scatter over the defect range may be rejected, at least for this phased array set-up. Both change of signal mean level and standard deviation will affect the estimation of $\operatorname{POD}(\mathrm{a})$. This illustrates a typical parameter issue that is very time consuming to conduct with simulated data and almost impossible with experimental data where meta-modeling may open great opportunities. With the meta-model developed in this work 5000 runs at each defect setting were emulated in a fraction of a second.

\section{THEORY}

\section{Simulation of Phased Array Ultrasonic Signal Amplitude}

The simulation engine used to investigate the procedure of phased array testing is simSUNDT [6]. It is a simulation environment for UT that has been modified for evaluation of the new UT testing technique phased array [7].

simSUNDT was delivered to the Swedish nuclear power industry at a workshop in Göteborg (2004). The simulation tool is freeware made available for all parties involved in testing activities at Swedish nuclear plants. simSUNDT is based on a mathematical kernel,

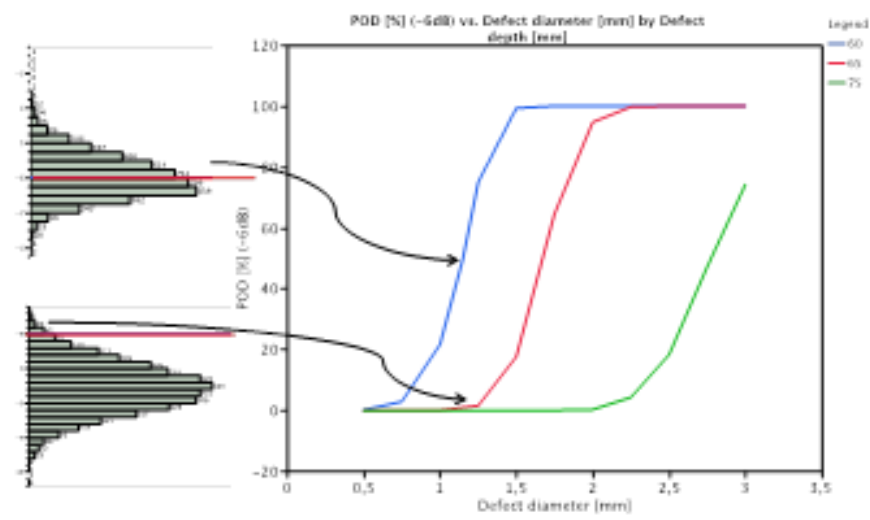

FIGURE 1. Synthetic $\operatorname{POD}(\mathrm{a})[\%]$ as a function of a - side drilled hole diameter [mm] for holes located at different depths [mm]. To the left and below two Monte Carlo simulated distributions exemplifying how the signal output varies at different side drilled hole diameter and depth depending on input parameter variation. The detection threshold or decision criterion is set to signal amplitude of $-6 \mathrm{~dB}$ relative a calibration signal. The relative part of the distribution above the threshold indicates the chance to detect such a hole. It is approximately a $50 \%$ chance to detect a hole with $1,15 \mathrm{~mm}$ diameter at $60 \mathrm{~mm}$ depth, whereas it is only $1,5 \%$ chance to detect a 1,25 diameter hole at $65 \mathrm{~mm}$ depth. 

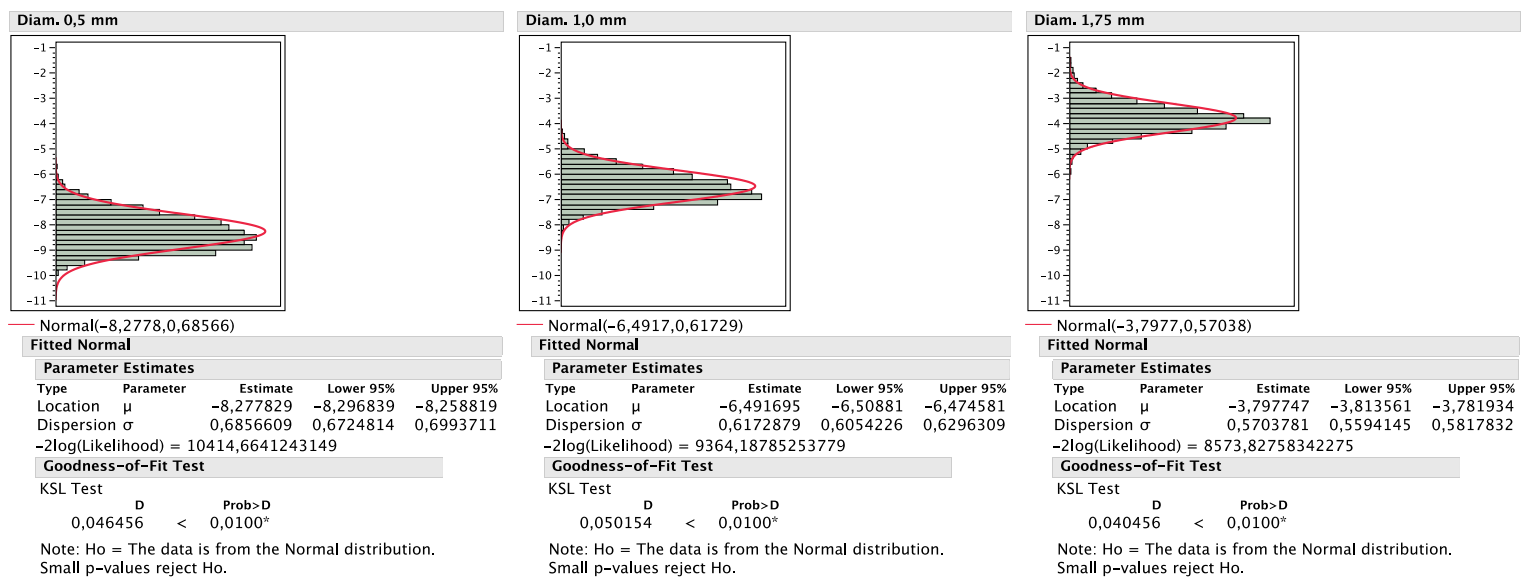

FIGURE 2. The three graphs above represents the output signal distribution (â) from a side hole diameters $0,5 \mathrm{~mm}, 1,0 \mathrm{~mm}$ and $1,75 \mathrm{~mm}$ at $60 \mathrm{~mm}$ depth (that is the defect is located $60 \mathrm{~mm}$ below the object surface). The same variations of input variables have been used, except defect diameter. The standard deviations of the output signal significantly vary with the size of the defect: $0,69 \mathrm{~dB}, 0,62 \mathrm{~dB}$ and 0,57 dB, respectively. Both mean level and the varying scatter will affect the proportion of the distribution above a certain threshold influencing on the POD curve slope.

field approach) and integral equations depending on defect type. The present version is limited to isotropic and homogeneous material. The limitation lies in the mathematical description of the defects that needs to be in a simple mathematical form (strip like, rectangular, circular, spherical, etc.).

The Windows-based software is made to resemble corresponding testing environment and commercial analysis tools available on the market. The output data is in a standard format. A noise model has been implemented in order to render realistic data with noise due to grain scatter. This, since one of the purposes of the software is to complement the use of test blocks.

An illustration of the modification of simSUNDT for phased array testing is shown in Fig. 3 and the modification is thoroughly described in [7]. The simulation model was verified with measurements on the sample shown in Fig. 4, with the experimental set-up, Fig. 5, at Siemens Industrial Turbomachinery in Finspång, Sweden, performed by Mattias Broddegård [8], as part of the Turbokraft project LIFE3. The corresponding for the zero degree probe is shown in Fig. 6. As can be deduced from the figure the simulations and the experimental data match very well both quantitatively (the maximum amplitude) and qualitatively (the energy divergence).

When it came to the angled probe situation it was more difficult to get the same good agreement though only in terms of energy divergence. This is due to the fact that the probe is modeled by a number of equi-sized boundary conditions on the scanning surface.

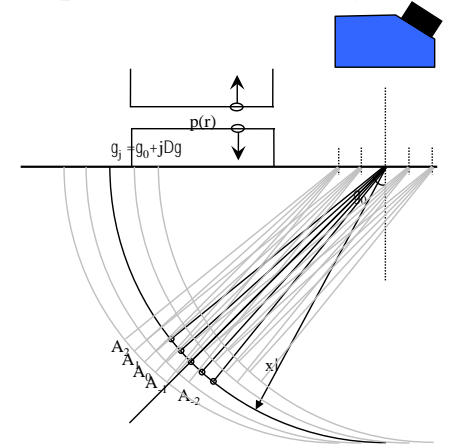

FIGURE 3. The geometry for the mathematic modeling of the phased array set-up shows that each element is prescribed as a boundary condition on the scanning surface that produces a plane wave in the far field with a specified angle. 


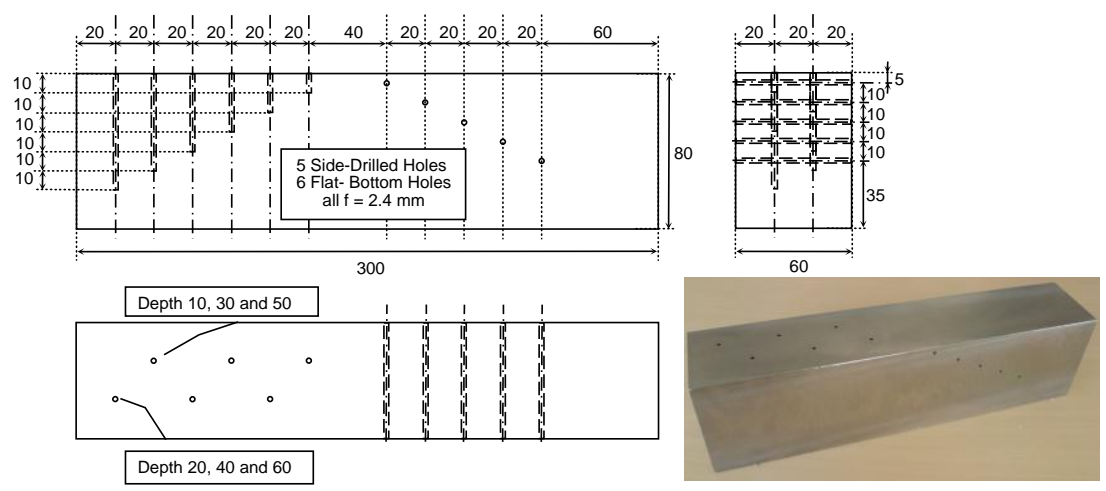

FIGURE 4. Dimensions of the test block $(12 \% \mathrm{Cr}$ steel $)$ with five side-drilled holes $(2.4 \mathrm{~mm})$ providing with ten different depths. Note that the depicted flat bottom holes in the test piece were excluded from the validation.
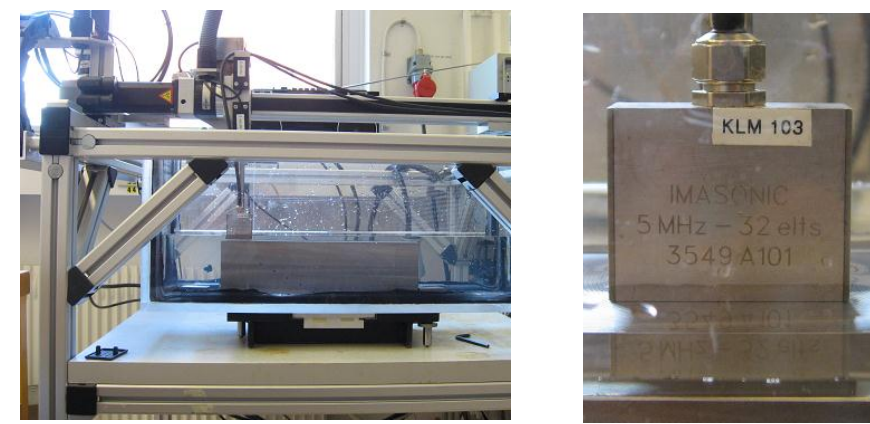

FIGURE 5. Measurements have been carried out with the Omniscan Phased Array system at the Materials laboratory at Siemens Industrial Turbomachinery using two different phased array probes scanning the test piece.

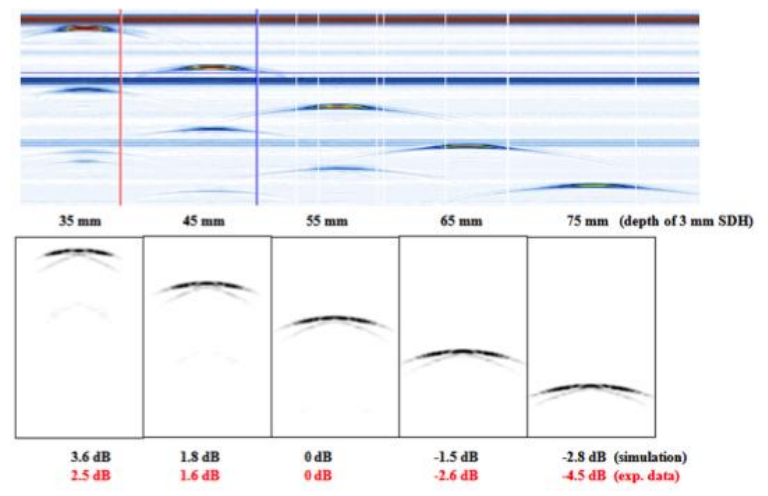

FIGURE 6. Qualitative comparison of the energy distribution between the probe (upper pictures) and tabled quantitative evaluation of the maximum signal responses.

Each of these elements is prescribed with an angle but in reality this angle is reached with a plastic wedge between the surface of the object and the un-angled phased array probe. Both the energy distribution and corresponding effective area of each element is prescribed to be equal in our model, which thus explains the qualitative discrepancy.

\section{EXPERIMENTS}

\section{Predictive Modeling (Meta-Modeling) of Signal Amplitude}

There are generally two main reasons for emulation of the simulator by metamodeling: first meta-modeling quickly increase the knowledge of how the system react to stimuli when input variable settings are altered, since the response is captured with a 


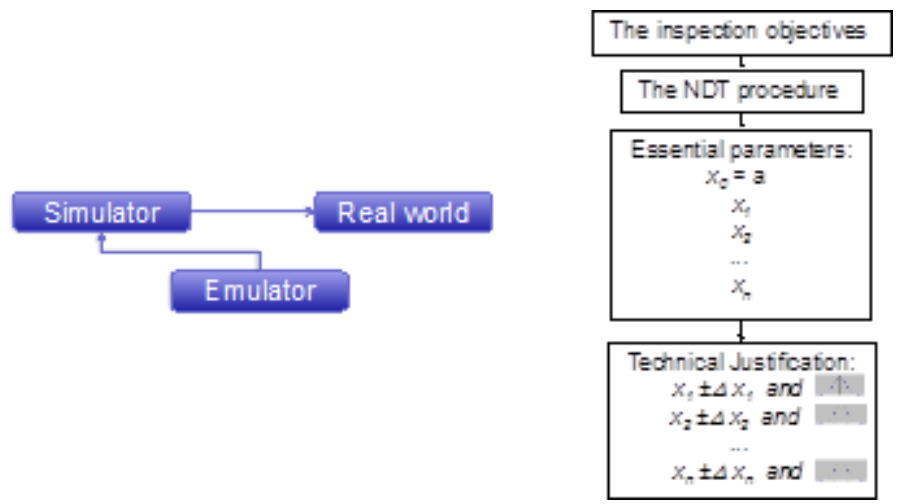

FIGURE 7. The meta-modeling of the emulator of the simulator of the real word build upon the identification of all relevant parameters in the experimental procedure.

simple regression model based on orthogonal arrays. Secondly, thanks to the simple regression model multi-parameter studies are readily done to a minimum of calculation time. These studies can include more parameters and resolve hidden relationships due to the fact that the input parameters can be varied independently, which is difficult or even impossible with real world measurements. The work path of the multi-parameter modeling building of the simulated phased array signal amplitude follows standard robust engineering stages:

1. Screening by fractional design of experiments (FroD), with the purpose to get rid of the least influential factors and lock them to the most favorable positions. Figure 7 show the procedure and table 1lists the essential parameters used and how the were locked or modeled at different stages of the process.

2. Full-factorial design of experiments (DoE), in order to sort out interactions, check for curvature and further reduce the number or influencing factors.

3. Response surface modeling by fitting a higher order polynomial model, to be used for three major purposes:

a. Aligning the emulator to the measurements, that is to find a setting for the set of simulator parameters in the emulator model that has limited connection to the experiment set-up for the verification measurements. And fine-tune the model to follow the measurement within the experimental range, illustrated in Fig. 8.

b. To build an emulator that predicts output signal amplitude as a function of defect depth and diameter, ultrasound damping, probe angle and elements in contact, Fig. 9. In this case the predictive modeling was done with the custom design algorithm in the statistical software package JMP8 from SAS Institute Inc and in a sequence of experimental designs were in total 47 runs used for modeling.

c. Use the meta-model with the parameters that are technical justified in order to introduce relevant variation from the input parameters for the Monte Carlo simulations of the signal output distribution shown in Fig. 10 as a function of defect depth and diameter.

\section{RESULTS}

\section{Emulation of Synthetic POD}

The synthetic POD as a function of defect size and depth emulated according to the above procedure are shown in Fig. 1. The $\operatorname{POD}(a)$ data points are generated as the 
TABLE 1. Essential parameters. The screening of the simulator started with 13 parameters. Five were locked due to limited impact on either the difference between simulator and measurements or the signal amplitude. Three were locked to minimize difference between simulator and measurements. And four technically justified were used for Monte Carlo simulations of output distributions.

\begin{tabular}{|c|c|c|c|c|c|c|}
\hline \multicolumn{4}{|c|}{ Simulation control factors } & \multirow{2}{*}{ Locked at } & \multirow{2}{*}{ Reason for locking } & \multirow{2}{*}{$\begin{array}{c}\text { Computer } \\
\text { experimental } \\
\text { stage }\end{array}$} \\
\hline Factor & unit & low & high & & & \\
\hline Focal plane & $\mathrm{mm}$ & 20 & 500 & 20 & Change scenario & \multirow{5}{*}{$\begin{array}{c}\text { Screening } \\
\text { in three steps } \\
\text { with fractional } \\
\text { design of } \\
\text { experiements }\end{array}$} \\
\hline Focal adjustment & $\%$ & -20 & 20 & 0 & \multirow{4}{*}{$\begin{array}{l}\text { minor impact } \\
\text { on both responses }\end{array}$} & \\
\hline Couplant & $\mathrm{mm}$ & 0,05 & 0,4 & 0,2 & & \\
\hline Sound velocity tranvers (T) & $\mathrm{mm} / \mathrm{s}$ & 5404 & 6604 & 6004 & & \\
\hline Sound velocity tranvers (S) & $\mathrm{mm} / \mathrm{s}$ & 2979 & 3641 & 3310 & & \\
\hline Frequence & $\mathrm{MHz}$ & 4 & 6 & 5,75 & \multirow{3}{*}{$\begin{array}{l}\text { Set to minimise difference } \\
\text { between measure and } \\
\text { simulated signal amplitude } \\
\text { (delta }=0 \text { ) for the defect depth } \\
\text { range tested - calibration of } \\
\text { simulation }\end{array}$} & \multirow{3}{*}{$\begin{array}{l}\text { Full factorial } \\
\text { design of } \\
\text { experiments }\end{array}$} \\
\hline Band width & $\mathrm{MHz}$ & 3 & 5 & 4 & & \\
\hline Sensor length & $\mathrm{mm}$ & 11 & 22 & 20 & & \\
\hline Defect depth & $\mathrm{mm}$ & 15 & 75 & \multirow{4}{*}{\multicolumn{3}{|c|}{$\begin{array}{l}\text { Control factors for emulation of the simulation by predictive } \\
\text { modelling: meta-modelling }\end{array}$}} \\
\hline Amplitude damping & $\%$ & 0 & 4 & & & \\
\hline Angle & & 41 & 49 & & & \\
\hline Sensor elements & \# & 2 & 20 & & & \\
\hline
\end{tabular}

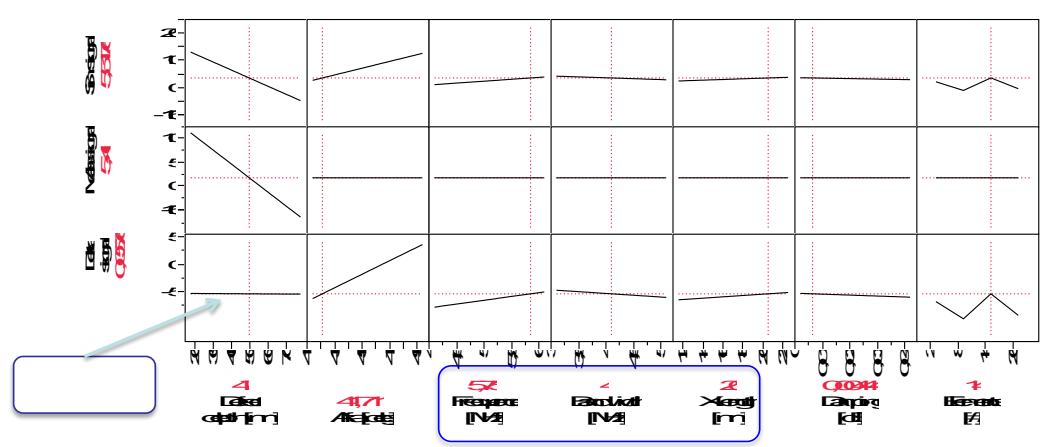

FIGURE 7. Show the modeling stage $3 \mathrm{a}$ - aligning the emulator to the measurements using parameters with limited impact on the signal amplitude. The purpose is to use the parameters with least impact on signal amplitude and adjust them such that the difference between measured and simulate is constant for all defect depths.
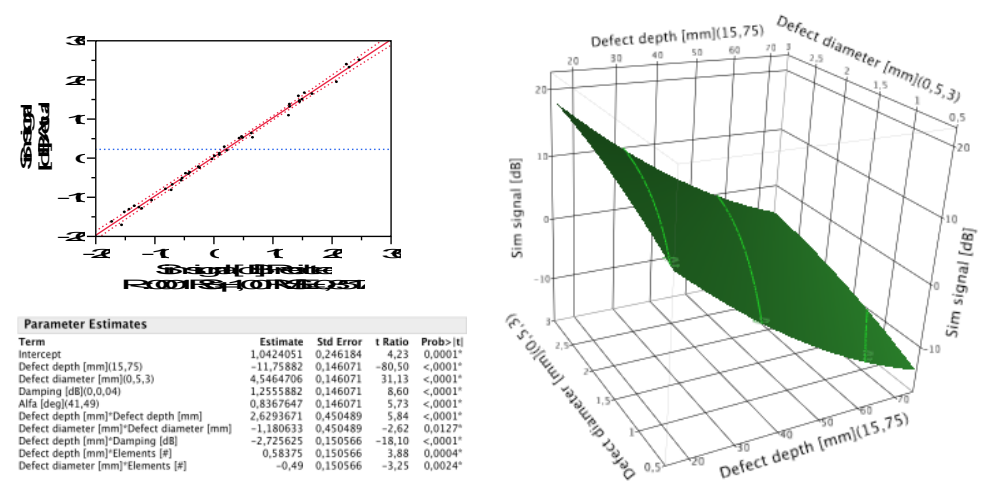

FIGURE 8. The meta-model (emulator) of the simulator signal amplitude response.

proportion of the emulated output distribution above the decision criteria at $-6 \mathrm{~dB}$ of the reference signal, according to standard calibration procedure for UT.

\section{DISCUSSION AND CONCLUSIONS}

- $\operatorname{sim}$ SUNDT has the possibility to simulate phase array, and is experimentally verified for the current setup for 0deg probe.

- $45 \mathrm{deg}$ probe require additional calibration procedure relative measurements in present stage of simSUNDT, due to the plastic wedge used to tilt the probe. 


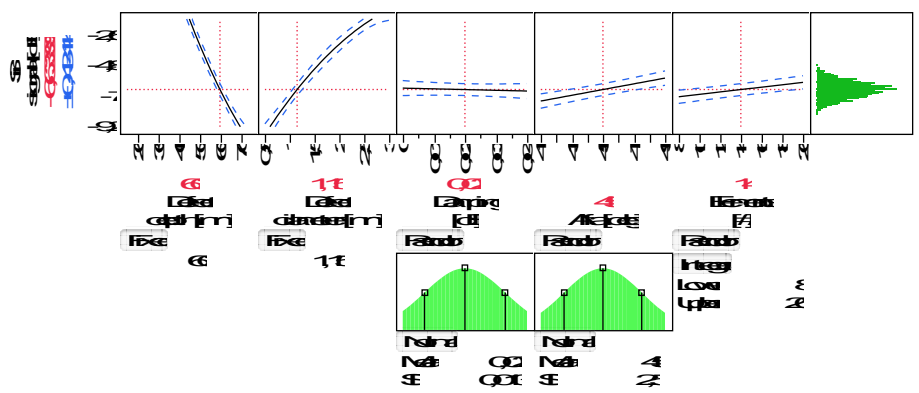

FIGURE 9. Monte Carlo simulation of the emulated signal amplitude output distribution at defect depth 60 $\mathrm{mm}$ and a defect diameter of $1,15 \mathrm{~mm}$. The variation on the damping represents microstructural changes in the material. The variation in probe angle and elements represents operation variations.

- The present methodology to emulate POD by meta-modeling (robust design methodology) works for proposed procedure for phased array testing. This procedure facilitates the understanding and development of testing procedures with a holistic application approach rather than a detailed mathematical modeling of the physical stages of the data collection process. The modeling can both be done with meta-modeling on simulations or predictive modeling from experiments based on design of experiments.

- The distribution of the output signal amplitude $[\mathrm{dB}]$ is skewed by response surface curvature, meaning that normally distributed input variation do not yield normally distributed output. An example of this shown in Fig. 2. Since the normal procedure for POD studies assume that the output scatter is independent of defect size and that $\hat{a}$-distribution is the same over the range of the defect size this procedure may be used to identify parameters influencing scatter and not only mean levels of NDT responses.

- The calculation time per data point is significantly reduced compared to the simulations $\sim 1: 10000$.

\section{ACKNOWLEDGEMENTS}

This research has been funded by the Swedish Energy Agency, Siemens Industrial Turbomachinery AB, Volvo Aero Corporation, and the Royal Institute of Technology through the Swedish research program TURBOPOWER, the support of which is gratefully acknowledged.

\section{REFERENCES}

1. B. Bergman and B. Klefsjö, Quality from customer needs to Customer Satisfaction, Third Edition, Studentlitteratur, Lund, ISBN 978-91-44-05942-6.

2. G. Persson, P. Hammersberg, and H. Wirdelius, POD Generated by Monto Carlo simulation using a meta-model based on the simSUNDT Software, QNDE proceedings, (2011).

3. MIL-HDBK-1823A, (2009), Nondestructive Evaluation System Reliability Assessment, Available for download at http://mh1823.com/mh1823.

4. ENIQ, (2010), Report No 41 Probability of detection Curves: Statistical Best-Practices, European Commosion Joint Research Centre, Institute for Energy, Petten: ENIQ.

5. A. P. Berens, NDE Reliability Data Analysis, Non-Destructive Evaluation and Quality Control: Qualitative Non-Destructive Evaluation, ASM Metals Handbook, 17, pp. 689-701, (1989).

6. A. Boström and H. Wirdelius, J. Acoust. Soc. Am., 97, 2836-2848, (1995).

7. G. Persson and H. Wirdelius, Review of progress in quantitative nondestructive evaluation, 29, 1211, pp. 2125-2132, Melwill, NY: AIP Conference Procedings, (2010).

8. M. Broddegård, Turbogkraft project LIFE3, Phased Array measurement results, Siemens Industrial Turbomachinery AB, (2009). 